\title{
Um Modelo Iterativo para o Ensino de Engenharia de Software Baseado em Abordagens Focadas no Aluno
}

\author{
Carlos S. Portela $^{1}$, Alexandre M. L. Vasconcelos ${ }^{1}$, Sandro R. B. Oliveira ${ }^{2}$ \\ ${ }^{1}$ Centro de Informática - Universidade Federal de Pernambuco (UFPE) \\ Caixa Postal 7851 - 50.740-560 - Recife - PE - Brasil \\ ${ }^{2}$ Programa de Pós-Graduação em Ciência da Computação - UFPA \\ Belém - PA - Brasil \\ $\{$ csp3,amlv\}@cin.ufpe.br, srbo@ufpa.br
}

\begin{abstract}
The Software Engineering (SE) discipline has a wide amount of topics and theoretical concepts that are best learned in practice. However, most teachers adopt traditional approaches to teach, such as expositive lessons. Through this context, this paper presents an iterative model based on the main approaches, methods and techniques focused on the student that are applied in the SE teaching. A specialist's panel composed of 3 (three) PhDs who act as teachers/researchers in the SE teaching area, did the evaluation of the structure and usability of this model. This panel considered the model complete and consistent. From the application of this model, we expect that students develop technical skills in SE at the application level.
\end{abstract}

Resumo. A disciplina de Engenharia de Software (ES) possui uma ampla quantidade de tópicos teóricos que é melhor fixada na prática. No entanto, a maioria dos professores adotam abordagens tradicionais de ensino, como aulas expositivas. Mediante este contexto, este artigo apresenta um modelo iterativo baseado nas principais abordagens focadas no aluno que são aplicadas no ensino de ES. A avaliação deste modelo foi feita através de um painel de especialistas composto por 3 (três) professores/pesquisadores na área de ES. Este painel de especialistas considerou o modelo completo e consistente. A partir do uso deste modelo, espera-se que os alunos desenvolvam competências técnicas em ES no nível de aplicação.

\section{Introdução}

A Engenharia de Software (ES) constitui-se como uma das disciplinas de maior relevância na área da Computação [Ohlsson e Johansson 1995]. De acordo com a ACM/IEEE (2014), seu objetivo consiste na aplicação de teoria, conhecimento e prática para o desenvolvimento efetivo e eficiente de sistemas de software que satisfaçam os requisitos dos usuários. Neste sentido, a ES possui muitos conceitos teóricos que são melhores fixados através da sua aplicação prática. No entanto, nem todos os cursos oferecem essa oportunidade aos alunos de realizarem atividades práticas [Marques, Quispe e Ochoa 2014]. Segundo Prikladnicki et al. (2009), muitos professores adotam abordagens tradicionais para ensinar ES, como aulas expositivas, que acabam sendo pouco eficientes, pois são centradas apenas no professor. 
Existe um consenso entre os pesquisadores da área de que abordagens práticas são as mais indicadas para o ensino de Engenharia de Software [Portela, Vasconcelos e Oliveira 2016]. Adicionalmente, alguns autores destacam que esse ensino deve ser mais centrado no aluno, a fim de aumentar sua motivação, participação e, consequentemente, sua aprendizagem [Prikladnicki et al. 2009].

Mediante o contexto exposto, este artigo apresenta um modelo iterativo baseado nas principais abordagens, métodos, estratégias e técnicas focadas no aluno que são aplicadas no ensino de ES. Não é pretensão dos autores criar um novo modelo de ensino, mas sim integrar as abordagens práticas identificadas na literatura em um único modelo iterativo, a fim de estabelecer um ciclo de aprendizagem [Kolb 1984] e contemplar os diferentes estilos de aprendizagem dos alunos [Fleming e Mills 1992].

Além desta seção introdutória, a Seção 2 apresenta a fundamentação teórica deste trabalho, destacando os métodos de ensino focados no aluno, práticas de capacitação da indústria e teorias e estilos de aprendizagem. A metodologia de pesquisa é descrita na Seção 3, a partir das etapas e métodos adotados. A Seção 4 apresenta o modelo iterativo de ensino proposto, destacando a aplicação das abordagens de ensino selecionadas. A avaliação do modelo, a partir de um painel de especialistas, é descrita na Seção 5, bem como os seus resultados. Por fim, as conclusões e os trabalhos futuros são apresentados na Seção 6.

\section{Fundamentação Teórica}

Diversos autores analisaram abordagens de ensino e aprendizagem existentes a partir de seus princípios, dos componentes necessários ao fenômeno educativo e de seus efeitos sobre o indivíduo e a sociedade. Dentres estes, destaca-se o trabalho de Mizukami (1986), que nomeia as diferentes abordagens do processo de ensino e aprendizagem, sendo estas: Tradicional; Comportamentalista; Humanista; Cognitivista; Sociocultural.

A partir da análise das características de cada uma destas abordagens, observouse que a Abordagem Humanista é predominante no modelo de ensino apresentado neste artigo. Esta predominância dá-se em função do foco do modelo no sujeito, onde o ensino deve ser "centrado no aluno". Outra característica desta abordagem no modelo diz respeito ao papel do professor, que deve atuar como um "facilitador da aprendizagem", ou seja, deve fornecer condições para que os alunos aprendam, devendo tomar atitudes favoráveis e condizentes com essa função [Mizukami 1986].

No entanto, Mizukami (1986) enfatiza que a Abordagem Humanista não contempla técnicas ou métodos para facilitar a aprendizagem. Uma das contribuições do modelo de ensino aqui apresentado é a sugestão de abordagens de ensino que proporcionam um aumento do engajamento e da motivação dos alunos. Como diferencial, este modelo incorpora práticas de capacitação adotadas pela indústria de software, como Coaching e Mentoring [Gomes et al. 2015]. Estas práticas permitem aos profissionais alvos da aplicação destas tanto a aquisição de novas competências quanto o desenvolvimento das já existentes. Desta maneira, pretende-se que os alunos possam desenvolver competências técnicas em nível de aplicação do conhecimento, de forma similar ao nível requerido para um profissional iniciante na área de Engenharia de Software. As subseções a seguir apresentam as principais abordagens práticas adotadas no modelo proposto. 
VI Congresso Brasileiro de Informática na Educação (CBIE 2017)

Anais do XXVIII Simpósio Brasileiro de Informática na Educação (SBIE 2017)

\subsection{Realização de Projetos Práticos}

O desenvolvimento de software requer muitas habilidades, como definição do projeto, gerenciamento, programação, validação, análise, estudo dos usuários, documentação, integração de sistemas e técnicas específicas. De maneira geral, os projetos práticos de cursos de graduação em Computação são aplicados a fim de preparar os estudantes para a prática profissional [Gnatz et al. 2003] [ACM/IEEE 2014]. Nestes projetos, tradicionalmente, os estudantes trabalham em grupos para desenvolver um software em reposta a um problema do mundo real.

Ohlsson e Johansson (1995) defendem que a realização de um projeto prático de desenvolvimento permite que o graduando aprenda a gerenciar o tempo, prioridade e progressos. Outros benefícios da realização destes projetos incluem o trabalho em grupo, a avaliação de potenciais soluções, o desenvolvimento de habilidades de comunicação e de relações interpessoais [ACM/IEEE 2014].

\subsection{Aprendizagem Baseada em Problemas}

Segundo Bessa, Cunha e Furtado (2012), existe um método utilizado na educação médica, desde a década de 70, denominado Problem Based Learning (PBL), que tem sido adaptado para diferentes áreas de atuação e diferentes níveis educacionais. De maneira geral, PBL pode ser definido como um método instrucional que usa um problema para iniciar, direcionar e motivar o aprendizado. De maneira especifica, PBL é um método que preza pelo uso de problemas baseados no mundo real para estimular os alunos a desenvolverem o pensamento crítico, habilidades para solução de problemas e adquirirem conhecimento [Andrade et al. 2010].

Embora concebido para o ensino da medicina, o método PBL vem sendo utilizado em outras áreas, como na Engenharia de Software [Andrade et al. 2010]. Esta aplicação na ES dá-se por meio de um modelo adaptado, de forma parcial, dentro do currículo convencional, ou em partes de uma disciplina. Bessa, Cunha e Furtado (2012) ressaltam três importantes princípios do método PBL que podem auxiliar o ensinoaprendizagem de Engenharia de Software: (1) o aprendizado acontece em um ambiente onde os estudantes estão imersos na prática, em atividades em que recebem feedback de seus colegas estudantes e professores; (2) os estudantes recebem guias e suporte de seus pares, de maneira a promover um ensino multidirecional envolvendo outros estudantes, professores e monitores; e, (3) o aprendizado é funcional, a partir de problemas reais.

\subsection{Uso de Jogos e Simuladores}

Jogos estão cada vez mais presentes como uma prática habitual no ensino, sendo concebidos como uma atividade motivadora no processo de ensino-aprendizado [Monsalve, Werneck e Leite 2010]. De acordo com Savi (2011), estes tipos de jogos possuem objetivos educacionais definidos, sendo projetados especificamente para ensinar determinados temas ou reforçar e apoiar a aprendizagem de habilidades.

Além dos jogos educacionais, destacam-se as ferramentas de simulação que, geralmente, simulam um contexto de uma fábrica de software, instituição de ensino e/ou empresa e seus respectivos participantes, onde cada estudante deve assumir um papel e desempenhar atribuições específicas nos seus ambientes [Bessa, Cunha e Furtado 2012]. 
VI Congresso Brasileiro de Informática na Educação (CBIE 2017)

Anais do XXVIII Simpósio Brasileiro de Informática na Educação (SBIE 2017)

Estas ferramentas permitem o desenvolvimento de experiências existentes no mundo real, reduzindo assim as lacunas existentes entre teoria e prática.

\subsection{Discussão de Casos Práticos}

Essa abordagem consiste basicamente na apresentação de relatos de experiências da indústria e na discussão dos problemas e soluções encontrados. A maioria dos relatos de experiências dá-se através de estudos de casos. De acordo com Marques, Quispe e Ochoa (2014), grande parcela das abordagens de ensino de ES identificadas em seu mapeamento sistemático trata da discussão de estudos de casos.

As diretrizes curriculares da ACM/IEEE (2014) enfatizam que a discussão de estudos de caso permite incorporar elementos do mundo real ao ensino e auxilia na efetividade do ensino de conceitos. Estes estudos expõem projetos e sistemas para que os alunos possam analisar criticamente e reusar as soluções propostas [ACM/IEEE 2014]. Assim, os alunos desenvolvem habilidades em pensamento analítico lendo e discutindo problemas complexos da vida real [Marques, Quispe e Ochoa 2014].

\subsection{Práticas de Capacitação da Indústria}

Os autores deste artigo realizaram um levantamento com 10 especialistas da área (consultores e implementadores de modelos de qualidade que também atuam como professores de graduação) a fim de identificar quais as práticas adotadas na capacitação de profissionais. A partir da análise das respostas obtidas, observou-se que a prática de capacitação mais adotada foi mentoring, que consiste no aconselhamento oriundo de indivíduos que detêm maior conhecimento e experiência que outros [Gomes et al. 2015]. A segunda prática mais adotada foi workshop, onde os consultores realizam um seminário de curta duração, apresentando técnicas e métodos e demonstrando como estes podem ser aplicados.

O uso de dinâmicas, onde um instrutor sugere uma atividade lúdica análoga a uma atividade técnica, e a prática de coaching, onde um profissional estimula e inspira outro a maximizar seu potencial, por meio do desenvolvimento de novos e mais efetivos comportamentos [Gomes et al. 2015], ainda são poucos explorados por consultores. Destaca-se a necessidade da adoção destas práticas, devido aos benefícios inerentes à implementação das mesmas.

\subsection{Ciclo e Estilos de Aprendizagem}

A teoria de aprendizagem experimental de Kolb (1984) define um ciclo de aprendizagem, conforme apresenta a Figura 1. Segundo Kolb (1984), para aprender efetivamente, devese planejar e praticar o que se pretende aprender (Experimentação Ativa). Em seguida, deve-se fazer algo, ou seja, ter uma experiência (Experiência Concreta). Após esta experiência concreta, o aluno deve revisar e refletir sobre o que foi feito (Observação Reflexiva). Por fim, deve-se realizar uma conclusão sobre o aprendizado obtido a partir da experiência (Conceptualização Abstrata). Contudo, a aprendizagem efetiva somente ocorre quando o aprendiz está habilitado para executar estes 4 (quatro) estágios. Este ciclo é a base da iteração do modelo de ensino apresentado neste artigo, conforme mostra a Figura 2 na Subseção 4.2. 


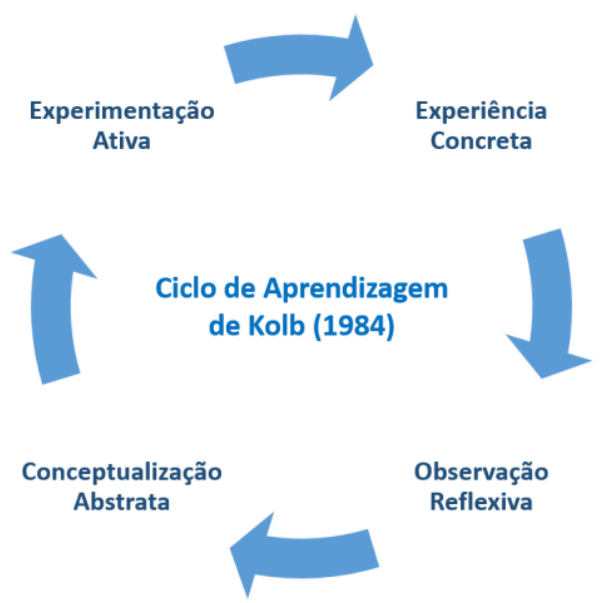

Figura 1. Ciclo de Aprendizagem de Kolb (1984)

A fim de operacionalizar o conceito de estilos de aprendizagem, através da preferência individual por diferentes tipos de estímulos sensoriais, Fleming e Mills (1992) propuseram o modelo VARK, acrônimo de Visual, Auditory, Read/Writing e Kinesthetic (em português; Visual, Auricular, Textual e Cinestésico). Cada um destes termos são categorias sensoriais que os seres humanos empregam no aprendizado e processamento de informações.

O modelo proposto incorpora abordagens de ensino que contemplam estes 4 (quatro) tipos de estímulos, por exemplo, através da realização de projetos práticos (cinestésicos), discussões (auditivos), uso de jogos (visuais) e leitura de artigos (textuais). Desta forma, pretende-se atingir uma ampla variedade de estilos de aprendizagens, pois a aplicação de um estímulo predominante poderia beneficiar apenas um subconjunto dos alunos e prejudicar outro, em função dos diferentes estilos de aprendizagem deles.

É importante destacar que essa abordagem de estilos de aprendizagem não é totalmente aceita pelos pesquisadores da área de ensino. De acordo com Paul (2017), há uma grande diferença entre a maneira como alguém prefere aprender e o que realmente leva a uma aprendizagem eficaz e eficiente. Adicionalmente, quase todos os estudos que relatam evidências de estilos de aprendizagem não conseguem satisfazer os critérios fundamentais para a validade científica. Apesar desta limitação, a adoção dessa abordagem no modelo não é determinante para a sua implementação, sendo adotado apenas para mapear as estratégias de ensino adotadas e os diferentes estilos descritos por Fleming e Mills (1992).

\section{Metodologia da Pesquisa}

Para atender os objetivos desta pesquisa, inicialmente analisou-se o currículo de referência da ACM/IEEE (2014) a fim de identificar os tópicos e as competências que os alunos devem desenvolver ao final da disciplina de ES. Em seguida, identificaram-se na Literatura as principais abordagens focadas no aluno para o ensino de ES (ver Subseções 2.1 a 2.4). Posteriormente, realizou-se um levantamento com especialistas sobre as principais estratégias de capacitação adotadas por estes na indústria para desenvolver competências profissionais (ver Subseção 2.5). 
Com base nestas abordagens e estratégias de ensino, considerando a Abordagem Humanista, o ciclo de aprendizagem de Kolb (1984) e os diferentes estilos de aprendizagem [Fleming e Mills 1992], definiu-se o modelo iterativo para o ensino de Engenharia de Software apresentado neste artigo.

A avaliação da estrutura e usabilidade deste modelo foi feita através de um painel de especialistas composto por 3 doutores que atuam como professores/pesquisadores na área de ensino de ES em diferentes instituições de ensino. Inicialmente, houve uma análise e avaliação individual do modelo (via questionário no Google Docs). Em seguida, foi feita uma reunião de consenso (via conferência no Skype) entre os especialistas, onde os mesmos justificaram as notas atribuídas na avaliação. Esta reunião buscou resolver as discordâncias, possibilitando o desenvolvimento de um consenso final do painel de especialistas. Como resultado, foi emitido um parecer conclusivo da avaliação.

\section{Modelo Iterativo para o Ensino de Engenharia de Software}

De acordo com Joyce e Weil (1972), um modelo de ensino pode ser definido como "um padrão ou plano que pode guiar a definição de currículos ou cursos, auxiliar a seleção de materiais de instrução e orientar as ações de um professor".

Joyce, Weil e Calhoun (2015) examinaram diversos modelos de ensino, a fim de categorizar os componentes individuais destes modelos. Como resultado, identificaram 6 (seis) componentes principais, os quais nortearam os componentes que compõem o modelo proposto e que serão apresentados nas subseções a seguir.

\subsection{Foco}

O foco descreve a intenção central do modelo, o objetivo do ensino. Os elementos da sintaxe giram em torno do objetivo principal do modelo. $\mathrm{O}$ modelo proposto fundamenta-se em abordagens práticas de ensino, portanto seu foco é na realização de atividades, a fim de desenvolver competências técnicas em ES nos alunos. A partir deste foco, recomenda-se esse modelo para professores que possuem um perfil de facilitador do ensino.

Não é foco deste modelo a definição dos conteúdos de ensino, pois na abordagem humanista estes são vistos como secundários no processo de ensino e aprendizagem [Mizukami 1986]. De forma similar, este modelo não foca no processo de avaliação de aprendizagem, pois a avaliação não se caracteriza, diretamente, como um dos componentes básicos de um modelo de ensino [Joyce, Weil e Calhoun 2015].

\subsection{Sintaxe}

A sintaxe descreve a estrutura do modelo através dos elementos de ensino. Inclui a sequência de passos envolvidos na organização do modelo. Neste sentido, o modelo é centrado na leitura de artigos técnicos com relatos de experiências, combinando aprendizagem centrada em problema (PBL), discussão de casos práticos, uso de jogos, simuladores ou dinâmicas, realização de projeto prático e reflexão. Sendo assim, este modelo permite apresentar, praticar e aplicar os tópicos das unidades de ES a fim de proporcionar uma aprendizagem mais contextualizada para os alunos e, consequentemente, desenvolver as competências técnicas necessárias para os graduandos ingressarem no mercado de trabalho. 
VI Congresso Brasileiro de Informática na Educação (CBIE 2017)

Anais do XXVIII Simpósio Brasileiro de Informática na Educação (SBIE 2017)

A sua aplicação é flexível, sendo possível adotá-lo tanto para o ensino de uma unidade de conhecimento específica quanto para uma disciplina de ES completa. A Figura 2 apresenta as 6 (seis) etapas deste modelo e o fluxo de iteração entre elas.

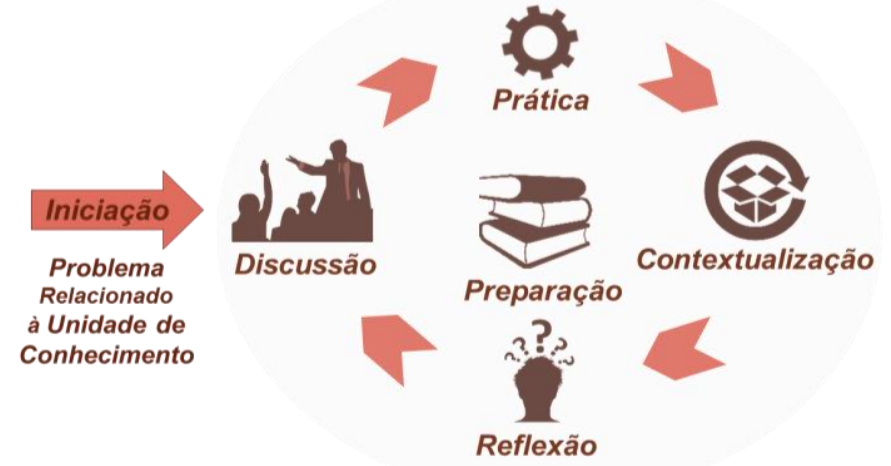

Figura 2. Modelo Iterativo para o Ensino de Engenharia de Software

O estudo de cada unidade de conhecimento inicia-se a partir da identificação de um problema, relacionado ao projeto a ser desenvolvido. Então, os alunos realizam leituras de artigos técnicos (estímulo Textual) e assistem palestras (estímulo Auricular) relacionadas a estas unidades para criar consciência e compreensão. Em seguida, colocam os conceitos de aula em prática através de dinâmicas de grupo ou jogos (estímulo Visual). Posteriormente, os alunos integram as habilidades adquiridas durante as etapas anteriores no projeto prático (estímulo Cinestésico) da disciplina de ES. Por fim, os alunos refletem sobre essa rápida experiência de aprendizagem, se envolvendo em grupos de discussão sobre as lições aprendidas. Este ciclo iterativo deve ser executado para cada unidade de conhecimento da ES.

\subsection{Sistema Social}

O sistema social descreve a relação entre o professor e os alunos, bem como o papel desempenhado por cada um nas atividades que definem a natureza do sistema social. Diz respeito às funções interativas e relações entre o professor e o aluno e quais os comportamentos dos alunos devem ser recompensados.

No modelo proposto, o aluno é o centro do processo de ensino-aprendizagem, devendo assumir um perfil participativo, sendo um agente ativo neste processo. Para tal, deve buscar resolver os problemas propostos pelo professor de forma criativa e original através da aplicação de conhecimento na realização das atividades práticas. O professor, por sua vez, deve projetar situações de aprendizagem e atividades que estimulem e desafiem os alunos. Neste sentido, sugere-se que o professor realize atividades de coaching. Esta prática sugere a definição de objetivos estimulantes, o estabelecimento de metas desafiadoras, o respeito às diferenças individuais, o fornecimento/recebimento de feedback e o apoio na realização das atividades [Gomes et al. 2015].

A fim de aconselhar, inspirar e motivar os alunos, sugere-se que o professor convide um aluno egresso da instituição (ou profissional) que atue profissionalmente na área relacionada à unidade de conhecimento ministrada. Este profissional pode atender estes objetivos através de um processo semelhante ao mentoring. Esse processo sugere o uso de práticas como metáforas, analogias, histórias de personagens de sucesso, exemplos, demonstrações, relatos de experiência, dentre outras [Gomes et al. 2015]. 
VI Congresso Brasileiro de Informática na Educação (CBIE 2017)

Anais do XXVIII Simpósio Brasileiro de Informática na Educação (SBIE 2017)

\subsection{Princípios de Reação}

Os princípios de reação especificam ao professor como lidar com o aluno e como reagir às respostas destes durante o uso do modelo. As respostas na utilização de um modelo designado devem ser apropriadas e coerentes com o foco do mesmo. As práticas e métodos de ensino adotados em cada etapa do modelo, que especificam os princípios de reação e interação que permitem direcionar o foco do processo de ensino-aprendizagem para o aluno, são: PBL e Coaching na Fase de Iniciação; Mentoring na Fase de Discussão; Workshop e Dinâmicas na Fase de Prática; Coaching na Fase de Contextualização; Mentoring e Coaching na Fase de Reflexão.

\subsection{Sistema de Apoio}

O sistema de apoio define as condições de apoio necessárias para implementar o modelo com sucesso. Este apoio inclui requisitos de materiais de referência, permissões, instalações de equipamentos, laboratórios ou ambiente de aprendizagem que precisam ser acessados na utilização do modelo.

Quanto às habilidades, o modelo recomenda que o professor deve ter interesse no uso de abordagens práticas de ensino focadas no aluno e buscar identificar e considerar os diferentes tipos de aprendizagens dos alunos no processo de ensino-aprendizagem. Quanto ao material instrucional, o modelo possui exemplos de materiais (artigos, jogos, dinâmicas, etc.) de acordo com as unidades da ES. Os equipamentos dizem respeito aos requisitos necessários para instalar os jogos e simuladores, executar as vídeo-aulas e realizar o projeto prático da disciplina. Por fim, quanto aos ambientes de aprendizagem, recomenda-se um Laboratório de Informática, com Internet, uma biblioteca que contenha livros e revistas atualizadas, além da sala de aula.

\subsection{Aplicação e Efeitos}

A aplicação e efeitos especifica como os alunos podem aplicar o que o modelo ensina. Essa aplicação diz respeito à utilidade do modelo, uma vez que pode ser transferida para outras situações e experiências. O modelo de ensino proposto busca desenvolver conhecimento nos alunos através da leitura de artigos, vídeo-aulas e discussões relacionadas às unidades de conhecimento da ES. A partir destas práticas, o modelo contempla o nível "Conhecer", onde o aluno deve ter a capacidade de lembrar do conteúdo discutido nas etapas iniciais do modelo, Iniciação e Discussão.

Esse conhecimento prévio pode ser transferido para outras situações na próxima etapa do modelo, denominada Prática. Essas experiências de aprendizagem permitem ao modelo contemplar o nível "Compreender", onde o aluno deve ter a capacidade de entender o significado do conteúdo estudado. Em seguida, na etapa de Contextualização, os alunos podem aplicar o conhecimento adquirido durante a execução do projeto prático. Desta forma, o modelo contempla o nível "Aplicar", onde o aluno deve ter a capacidade de usar o conhecimento adquirido em situações práticas.

\section{Avaliação do Modelo}

Conforme descrito na Seção 3, a avaliação do modelo se deu através de um painel de especialistas composto por 3 professores/doutores com uma média de 8 anos de experiência docente na área de ES. Esse painel avaliou diversos critérios, relacionados à 
documentação e usabilidade do modelo, emitindo um parecer conclusivo ao final. $\mathrm{O}$ resultado completo deste processo está disponível em https://goo.gl/jS8j5R.

A partir da análise desta avaliação e do parecer final, conclui-se que painel de especialistas considerou o modelo completo e consistente. Os professores também destacaram a corretude, a flexibilidade e a utilidade do modelo para o ensino de ES. De acordo com o parecer de um avaliador, "o modelo é suficientemente sintético, e representativo do conjunto de teorias mencionadas na fundamentação. Se um professor não adere aos fundamentos do modelo, ele não é útil". Este avaliador respondeu que recomendaria, numa escala de 0 a 5 , fortemente (5) o uso do modelo. Já os outros 2 avaliadores destacaram que recomendariam largamente (4).

Como recomendações, os avaliadores citaram que devem ser melhorados a facilidade de uso e o conteúdo de apoio. Quanto a estes itens, encontra-se em desenvolvimento um sistema web a fim de melhorar a usabilidade do modelo e disponibilizar o material de apoio, bem como permitir ao professor submeter seu próprio material de aula de maneira colaborativa.

\section{Conclusões}

O modelo de ensino apresentado integra abordagens de ensino focadas nos alunos e adapta estratégias de capacitação da indústria para o contexto acadêmico. Adicionalmente, o modelo incorpora diversos estímulos de aprendizagem (cinestésico, auditivo, visual e textual). Assim, a principal contribuição deste modelo é abranger diferentes abordagens de ensino e estilos de aprendizagens a fim de desenvolver, de maneira mais adequada, competências técnicas para que os alunos possam atuar na área de ES.

Baseado nas principais funções de um modelo de ensino identificadas por Joyce, Weil e Calhoun (2015) e a partir da aplicação desse modelo em sala de aula, espera-se obter os seguintes resultados:

- Professores orientados na seleção de técnicas, estratégias e métodos de ensino adequados para motivar e engajar os alunos;

- Materiais instrucionais que sejam fontes de aprendizagem diferenciadas e eficazes;

- Alteração da atual dinâmica de ensino de ES através da adoção de abordagens focadas no aluno e práticas de capacitação da indústria;

- Melhoria do desenvolvimento de competências técnicas no ensino de ES em nível de aplicação de conhecimento.

Embora um painel de especialistas possa permitir uma análise profunda de um modelo, a validade dos seus resultados depende de uma indução empírica mais ampla. Sendo assim, como trabalhos futuros, serão realizados experimentos controlados para uso do modelo em sala de aula, a fim de verificar se os resultados obtidos reforçarão ou refutarão os resultados apresentados neste artigo. Adicionalmente, encontra-se em desenvolvimento um sistema web a fim de melhorar a usabilidade do modelo e disponibilizar um ambiente colaborativo para troca de materiais de apoio (artigos, vídeoaulas, dinâmicas, jogos, dentre outros) ao ensino de Engenharia de Software. 
VI Congresso Brasileiro de Informática na Educação (CBIE 2017)

Anais do XXVIII Simpósio Brasileiro de Informática na Educação (SBIE 2017)

\section{Referências}

ACM/IEEE (2014). Software Engineering 2014: Curriculum Guidelines for Undergraduate Degree Programs in Software Engineering.

Andrade, A. et al. (2010). "Aplicação do Método PBL no Ensino de Engenharia de Software: Visão do Estudante". ERBASE - Escola Regional de Computação dos Estados da Bahia, Alagoas e Sergipe.

Bessa, B.; Cunha, M.; Furtado, F. (2012) "ENGSOFT: Ferramenta para Simulação de Ambientes Reais para auxiliar o Aprendizado Baseado em Problemas (PBL) no Ensino de Engenharia de Software". Anais do XX Workshop sobre Educação em Informática. Curitiba-PR.

Fleming, N. D.; Mills, C. (1992) "Not Another Inventory, Rather a Catalyst for Reflection". To Improve the Academy, v. 11, Cap. 1, p. 137.

Gnatz, M. et al. (2003) "A Practical Approach of Teaching Software Engineering". Proceedings of the 16th Conference on Software Engineering Education and Training (CSEET'03). Madrid: IEEE, p. 120-128.

Gomes, A. et al. (2015), Coaching e Mentoring. 1 $^{\text {a }}$ ed. Rio de Janeiro: Editora FGV.

Joyce, B.; Weil, M. (1972), Models of Teaching. 1st. ed. Prentice-Hall.

Joyce, B.; Weil, M.; Calhoun, E. (2015), Models of Teaching. 9th. ed. Boston Pearson.

Kolb, D. (1984), Experiential Learning: Experience as the Source of Learning and Development. NJ: Prentice-Hall.

Marques, M.; Quispe, A.; Ochoa, S. (2014) "A Systematic Mapping Study on Practical Approaches to Teaching Software Engineering". Proceedings of the Frontiers in Education Conference. Madrid: IEEE, p. 1-8.

Mizukami, M. (1986), Ensino: As Abordagens do Processo. São Paulo: Epu.

Monsalve, E.; Werneck, V.; Leite, J. (2010) "SimulES-W: Um Jogo para o Ensino de Engenharia de Software". Anais do III Fórum de Ensino de Engenharia de Software (FEES). Salvador-BA.

Ohlsson, L.; Johansson, C. (1995). “A Practice Driven Approach to Software Engineering Education”. IEEE Transactions On Education, 291-295.

Paul A. (2017), Stop propagating the learning styles myth. Computers \& Education, Volume 106, Pages 166-171, ISSN 0360-1315.

Portela, C.; Vasconcelos, A.; Oliveira, S. (2016) "FRAMES: Uma Proposta de Framework para o Ensino de Tópicos da Engenharia de Software" Em Anais do XXVII Simpósio Brasileiro de Informática na Educação (SBIE 2016), p. 1361-1365.

Prikladnicki, R. et al. (2009) "Ensino de Engenharia de Software: Desafios, Estratégias de Ensino e Lições Aprendidas". Em Anais do II Fórum de Educação em Engenharia de Software. Fortaleza, Brasil.

Savi, R. (2011). Avaliação de Jogos voltados para a Disseminação do Conhecimento. Tese de Doutorado. Programa de Pós-Graduação em Engenharia e Gestão do Conhecimento: Universidade Federal de Santa Catarina, p. 236. 